\title{
Improving outpatient primary medication adherence with physician guided, automated dispensing
}

\author{
Jacob G Moroshek ${ }^{1,2}$ \\ 'Bioinformatics and Computational \\ Biology, ${ }^{2}$ Carlson School of \\ Management, University of Minnesota, \\ Minneapolis, MN, USA
}

This article was published in the following Dove Press journal:

ClinicoEconomics and Outcomes Research

5 January 2017

Number of times this article has been viewed
Background: Physician dispensing, different from pharmacist dispensing, is a way for practitioners to supply their patients with medications, at the point of care. The InstyMeds dispenser and logistics system can automate much of the dispensing, insurance adjudication, inventory management, and regulatory reporting that is required of physician dispensing.

Objective: To understand the percentage of patients that exhibit primary adherence to medication in the outpatient setting when choosing InstyMeds.

Method: The InstyMeds dispensing database was de-identified and analyzed for primary adherence. This is the ratio of patients who dispensed their medication to those who received an eligible prescription.

Results: The average InstyMeds emergency department installation has a primary adherence rate of $91.7 \%$. The maximum rate for an installed device was $98.5 \%$.

Conclusion: Although national rates of primary adherence have been found to be in the range of $70 \%$, automated physician dispensing vastly improves the rate of adherence. Improved adherence should lead to better patient outcomes, fewer return visits, and lower healthcare costs.

Keywords: automated dispensing, adherence, compliance, medication, physician dispensing, InstyMeds

\section{Background}

"Drugs don't work in patients who don't take them." [C. Everett Koop, MD, former Surgeon General]

Only an estimated $71.7 \%$ of new, written prescriptions are dispensed to patients. ${ }^{1}$ This is according to Fischer et al, who analyzed the difference between the number of prescriptions written electronically in two large populations of commercially insured outpatients in Massachusetts, and prescriptions actually filled at a pharmacy on the basis of insurance claims. In a large sample study of Quebec, Canada, Tamblyn et al found the rate of prescriptions filled to be only $68.7 \% .^{2}$ The behavior of not filling a prescription is known as primary medication nonadherence. The focus of this paper is measuring the rate of primary adherence for the system of automated dispensing technology, provided by InstyMeds Corporation, in the outpatient setting.

The World Health Organization defines medication adherence as "the degree to which the person's behavior corresponds with the agreed recommendations from a healthcare provider". ${ }^{3}$ However, many of the studies on adherence lack commonality in terms of how adherence is measured. Solomon and Majumdar view adherence as a broad process that begins with the receipt of the medication, continues with correct usage, and repeats with refills, if necessary. ${ }^{4}$ The focus of this paper is on primary
Correspondence: Jacob G Moroshek Carlson School of Management, University of Minnesota, Minneapolis, 321 19th Ave S, Minneapolis, MN 55455, USA Tel +l 9197244529 Email moro0027@umn.edu 
medication adherence in the emergency department (ED) or other outpatient setting. The definition of primary adherence that will be used here is similar to other research published on the subject: a prescription written by a provider, dispensed to a patient. ${ }^{1,2}$

Researchers have been studying adherence for decades. DiMatteo performed a meta-analysis on studies between 1948 and 1998 to measure long-term trends, finding that rates have not changed in the intervening years. ${ }^{5}$ The long period of study accentuates that this is a multifaceted challenge. In the ED, writes Yamamoto,

"Access to quality emergency care should be available to

all, including access to the essential components of after-

care. Failing to provide access to appropriate medications

compromises the emergency care safety net."

The best practice of providing access to medications remains most elusive in rural America. In many parts of the USA, patients drive distances exceeding 10 miles to get to a retail pharmacy. ${ }^{7}$ For these individuals, filling their first outpatient prescription can be a major challenge. Bissonnette et al found that pharmacy density in Oregon affected the rate of hospital admissions, with higher density areas having fewer readmissions. ${ }^{8}$

The primary medication nonadherence is not unique to rural America. Even in regions with higher population density, pharmacies often have limited hours of operation. Typical retail pharmacies for instance tend to operate 12 hours a day between $8 \mathrm{AM}$ and 8 PM, which leaves a large service gap of 12 hours. Patients falling outside these service hours are expected to go home, wait, and get their medication later. Several groups of patients are particularly challenged by having to travel to the pharmacy. Rittner and Kirk analyzed survey data of low-income elderly individuals, finding they rely heavily on public transportation. Each additional destination reduces the chance of receiving healthcare. ${ }^{9}$ Another at-risk group are patients with mental illness. Cramer and Rosenheck reviewed research on medication compliance in psychiatric treatment between 1975 and 1996 finding that patients with overlapping physical and psychiatric disorders were at very high risk for nonadherence. ${ }^{10}$ These are some of the factors contributing to the previously mentioned findings of Fischer et al that primary nonadherence in Massachusetts was almost $29 \%{ }^{1}$

Reduced adherence carries high economic costs. The IMS Institute for Healthcare Informatics calculates that improving medication adherence would eliminate at least $\$ 105$ billion in avoidable healthcare costs in the USA.${ }^{11}$ Poor outcomes from nonadherence can impact other economic factors, such as employee productivity. In a study of 10 employers with a total of 51,648 employee respondents, Loeppke et al found that health-related productivity losses are estimated to be 2.3 times higher than direct healthcare costs. ${ }^{12}$ Although these two studies are not specific to outpatient primary adherence, they do demonstrate the magnitude of the problem.

\section{InstyMeds background}

The goal of this study is to measure the rate of primary adherence for the automated dispensers installed by InstyMeds Corporation in the ED outpatient setting. ${ }^{13}$ InstyMeds was founded in 1999 with the goal of providing medications to patients at the point of care. The system falls under the branch of physician/practitioner dispensing, which differs from the more well-known pharmacist dispensing model. In a practitioner dispensing model, it is the practitioner's responsibility to perform the duties that are required of a pharmacist, such as patient counseling and drug-drug interaction checking. ${ }^{14}$ Proponents of physician dispensing cite better convenience, lower costs, higher adherence, and more information for patients. ${ }^{15}$ Criticism of physician dispensing has been that some practitioners profit from the markup on dispensed medications. ${ }^{16}$

Practitioner dispensing often involves multiple back-end obligations, such as inventory management, billing, regulatory reporting, purchasing repackaged wholesale medicine, labeling, and of course, dispensing. ${ }^{16}$ InstyMeds automates these operations. Practitioners offer patients the choice of a retail pharmacy or InstyMeds. If a patient chooses InstyMeds, they receive a printed document with not only medication information but also a code for retrieving their prescription. They proceed to the dispenser and follow touchscreen instructions. The patient's identity is verified along with insurance and billing prior to dispensing. A phone on the side of the dispenser allows for immediate connection to a 24-hour call center that can troubleshoot many issues. The practitioner has access to a cloud-connected interface to track whether patients obtained their medication along with other dispensing analytics.

Physician/practitioner dispensing regulations vary by state. InstyMeds, therefore, abides by the rules of the state in which the dispenser is located. Patient counseling is facilitated by the patient drug education information, which is printed with each medication order voucher from the automated dispensing software. Drug-drug interactions are also automatically detected by the practitioner's e-prescribing software, which is programmed to flag any potential interactions. The company owns several safety 
and design patents on its products. ${ }^{17}$ A 2013 study by Flynn tested InstyMeds dispensers at 41 locations in 12 states, for 1,001 total prescriptions dispensed. No dispensing errors of any type were detected. ${ }^{18}$

This study measures the rate of primary adherence using automated dispensing in the outpatient ED setting with InstyMeds installations. To accomplish this goal, the InstyMeds database of millions of prescriptions dispensed is used to collect and analyze data.

\section{Materials and methods}

A secure Microsoft SQL Server houses the database of InstyMeds dispensing and patient information. All HIPAA privacy standards were followed, and patient data were deidentified. Institutional Review Board approval was granted by the University of Minnesota.

Queries were run on the InstyMeds database to yield the following outputs:

1. Grand total of medications successfully dispensed from the start of database tracking from April 10, 2001 through June 3, 2016 at 19:15 GMT.

2. Total ED prescriptions voided from April 10, 2001 through June 3, 2016 at 19:15 GMT.

3. Total ED successful dispenses beginning July 22, 2011 through June 3, 2016 at 19:15 GMT.

4. Total ED prescriptions auto-voided and 48-hour voided beginning July 22, 2011 through June 3, 2016 at 19:15 GMT.

5. Total ED successful dispenses and auto-voids beginning July 22, 2011 through June 3, 2016 at 19:15 GMT at the facility with the highest ratio of successful to voided prescriptions.

When database utilization began in 2001, InstyMeds had three attributes applicable to adherence that were being tracked: "successfully dispensed", "changed to retail", and "voided". Prescriptions that were "changed to retail" were being sent to traditional retail pharmacies, instead of InstyMeds. Voided prescriptions were those that were cancelled for any reason, including when there were issues in processing insurance, when a facility was doing test dispenses, or when a patient failed to get the medication within 48 hours. Initially, the database did not specify the details of the void being used. That is to say, there was no way to tell the reason for the void that was recorded in the database.

This paper's working definition of primary medication adherence is a prescription written by a provider, dispensed to a patient. The rate of primary adherence, then, is found by taking successful dispenses divided by applicable written prescriptions. Non-applicable written prescriptions include:

1. When a patient elects to change their prescription to retail.

2. Voids due to facility test dispenses.

3. Voids due to insurance problems (subsequently handled on a custom basis by involving the practitioner).

Starting July 22, 2011, InstyMeds began tracking the reason for voided medications, including auto-voiding. An auto-void occurs when a patient does not dispense their prescription within 48 hours of the prescription being written. This meets the paper's working definition of primary medication nonadherence. The rate of primary medication adherence can, therefore, be calculated as:

\section{Successful dispenses \\ $\overline{\text { Successful dispenses + auto-voids }}$}

Another query is run to find the facility with the highest rate of primary medication adherence. This is to establish what the potential upper range is for facilities utilizing automated dispensing. Names have been removed to protect patient and customer confidentiality.

\section{Results}

Table 1 summarizes the results of the study. In $\sim 15$ years of operation, InstyMeds Corporation has dispensed almost three million prescription medications. Their rate of primary medication adherence in the ED, based on a sample size of $1,493,869$, is $91.7 \%$. However, the facility with the highest rate of primary medication adherence is over $98.5 \%$, with sample size $>36,000$ individuals.

\section{Discussion}

Describing the varieties of automated dispensers in 2001, Murray writes, "they are drug storage devices or cabinets that electronically dispense medications in a controlled fashion and track medication use." ${ }^{19}$ Almost all of the devices are

Table I The results of running queries on the InstyMeds SQL database

\begin{tabular}{lllll}
\hline Date range & Facility & Successful dispenses (A) & Voided or auto-voided (B) & Primary adherence rate A/(A+B) \\
\hline $04 / 10 / 2001-06 / 03 / 2016$ & All & $2,840,234$ & 371,298 voided & NA \\
$07 / 22 / 2011-06 / 03 / 2016$ & All & $1,493,869$ & 134,322 auto-voided & $91.7 \%$ \\
$07 / 22 / 2011-06 / 03 / 2016$ & Top performer & 35,946 & 539 auto-voided & $98.5 \%$ \\
\hline
\end{tabular}

Abbreviation: NA, not applicable. 
not patient facing, instead they assist nurses and pharmacists. ${ }^{20}$ InstyMeds differs from other automated dispensers for three reasons. 1) It falls under the branch of physician dispensing, a completely different set of laws and regulations from pharmacist dispensing. 2) InstyMeds is installed only in outpatient facilities. 3) The patient and dispenser interface directly.

Several types of automated dispensers have features similar to InstyMeds. The Asteres ScriptCenter MX kiosk, for instance, automates the task of payment and pickup for the patient, but pharmacists fill and check each prescription. ScriptCenter MX operates under the pharmacist dispensing model and has not published any findings on rates of primary adherence. ${ }^{21}$ The MedVantx MedStart Cabinet is an automated dispenser that interfaces only with the physician, who then hands the medication to the patient. The website claims a " $92 \%$ reduction in the number of patients who 'never' get their prescription". ${ }^{22}$ Finally, MedAvail Technologies' MedCenter offers an automated dispenser with services and features similar to those of InstyMeds. The company is running pilot programs in the USA and Canada, in which they claim improved primary drug adherence; however, the rates have not been published. ${ }^{23}$ The reason there is an absence of published research on rates of primary adherence with physician dispensing may be due to the fact that, when a physician manually dispenses directly to the patient, the primary adherence is automatically $100 \%$.

The previously cited rates of primary adherence suggest that $\sim 30 \%$ of patients do not receive their medication in the outpatient setting when sent to a retail pharmacy. ${ }^{1,2}$ This study, however, focuses on the outpatient ED population. That raises the question of whether patients experiencing acute medical problems will have a higher primary adherence than typical outpatients? In a study around patients with recent heart attacks, Jackevicius et al found that $\sim 25 \%$ of patients who suffered an acute myocardial infarction had not filled their prescriptions by 7 days post discharge. ${ }^{24}$ Tamblyn et al found that the odds of adherence were affected by the types of medication, age, copay, and the number of physician visits. ${ }^{2}$ These studies suggest that while rates of primary adherence do have variance, the outpatient population is universally challenged by nonadherence.

Another point of discussion is in understanding the difference between the average primary adherence of $91.7 \%$ and the maximum of $98.5 \%$. There might be several reasonable explanations. 1) Provider confidence makes a difference. When physicians have familiarity with the InstyMeds automated dispenser, they can more persuasively convince their patients to obtain their medications. 2) Long-term presence in a community. As patients have multiple exposures to the concept of automated dispensing, they become more familiar and comfortable with the device. In patient polling, 97.9\% of a sample of 6,449 respondents said they would use the dispenser again. A total of $95.0 \%$ of the respondents rated their overall experience as high. Having positive experiences with the technology means long-term adoption and higher rates of primary adherence.

\section{Conclusion}

Practitioners seeking to improve the primary medication adherence of their patients, until recently, had to either build an on-site pharmacy or absorb numerous back-end obligations of physician dispensing. These include inventory management, billing, regulatory reporting, purchasing repackaged wholesale medicine, labeling, and dispensing. InstyMeds is a unique solution as it automates these many obligations for the provider, while maintaining a high rate of adherence. This study found that InstyMeds' ED installations have an average primary adherence rate of $91.7 \%$ with an upper level at $98.5 \%$. Improved adherence over the national rate should lead to better patient outcomes, fewer return visits, and lower healthcare costs. Future research could seek to elucidate what factors enable some installations to have almost complete primary adherence with their InstyMeds dispensers.

\section{Acknowledgments}

The author thanks Brad Schraut for the tremendous support and the time taken to discuss the scale and scope of medication nonadherence. The author thanks Pratik Khetiya for helping to de-identify patient and customer data and for verifying the validity of database queries.

\section{Disclosure}

The author reports no conflicts of interest in this work.

\section{References}

1. Fischer MA, Stedman MR, Lii J, Vogeli C, Shrank WH, Brookhart MA, Weissman JS. Primary medication non-adherence: analysis of 195,930 electronic prescriptions. J Gen Int Med. 2010;25(4):284-290.

2. Tamblyn R, Eguale T, Huang A, Winslade N, Doran P. The incidence and determinants of primary nonadherence with prescribed medication in primary care: a cohort study primary nonadherence with prescribed medication in primary care. Ann Int Med. 2014;160(7):441-450.

3. Brown MT, Bussell JK. Medication adherence: WHO cares? Mayo Clin Proc. 2011;86(4):304-314.

4. Solomon MD, Majumdar SR. Primary non-adherence of medications: lifting the veil on prescription-filling behaviors. J Gen Int Med. 2010;25(4):280-281.

5. DiMatteo MR. Variations in patients' adherence to medical recommendations: a quantitative review of 50 years of research. Med Care. 2004;42(3):200-209. 
6. Yamamoto LG, Manzi S, Shaw KN, et al. Dispensing medications at the hospital upon discharge from an emergency department. Pediatrics. 2012;129(2):e562-e562.

7. Casey MM, Klingner J, Moscovice I. Pharmacy services in rural areas: is the problem geographic access or financial access? J Rural Health. 2002;18(3):467-477.

8. Bissonnette S, Goeres LM, Lee DSH. Pharmacy density in rural and urban communities in the state of Oregon and the association with hospital readmission rates. J Am Pharm Assoc. 2016;56(5):533-537.

9. Rittner B, Kirk AB. Health care and public transportation use by poor and frail elderly people. Soc Work. 1995;40(3):365-373.

10. Cramer JA, Rosenheck R. Compliance with medication regimens for mental and physical disorders. Psychiatr Serv. 1998;49(2):196-201.

11. Informatics. IIfH. Avoidable costs in US health care; 2013. Available from: http://www.imshealth.com/deployedfies/imshealth/Global/ Content/Corporate/IMS\%20Institute/RUOM-2013/IHII_Responsible_Use_Medicines_2013.pdf. Accessed June 20, 2015.

12. Loeppke R, Taitel M, Haufle V, Parry T, Kessler RC, Jinnett K. Health and productivity as a business strategy: a multiemployer study. J Occup Environ Med. 2009;51(4):411-428.

13. Available from: http://www.InstyMeds.com. Accessed June 1, 2016.

14. Milenkovich N. Physician in-office dispensing of Rx drugs. Drug Topics. 2014. Available from: http://drugtopics.modernmedicine.com/ drug-topics/content/tags/controlled-substances/physician-office-dis pensing-rx-drugs. Accessed June 1, 2016.
15. Perri M, III, Kotzan JA, Carroll NV, Fincham JE. Attitudes about physician dispensing among pharmacists, physicians and patients. $J \mathrm{Am}$ Pharm. 1987:NS27(10):57-61.

16. Meier B, Thomas K. Insurers pay big markups as doctors dispense drugs. The New York Times. 2012 July 12.

17. Rosenblum K. Automatic prescription drug dispenser. Google Patents. 2015.

18. Flynn EA. A Study of the Accuracy of InstyMeds. In press 2013.

19. Murray MD. Automated Medication Dispensing Devices. Purdue University School of Pharmacy; Rockville, MD: Agency for Healthcare Research and Quality; 2001.

20. Perini VJ, Vermeulen LC Jr. Comparison of automated medicationmanagement systems. Am J Hosp Pharm. 1994;51(15):1883-1891.

21. ScriptCenter MX. Available from: http://www.asteres.com/script center-mx/. Accessed June 1, 2016.

22. Increase medication adherence and improve patient satisfaction with the program that provides your patients with generic, brand and OTC medication samples at the point-of-care. Available from: http://www. medvantx.com/medvantx-for-you/healthcare-providers. Accessed June 1, 2016.

23. The MedCenter Remote Dispensing System. Available from: http:// www.medavail.com/medcenter0.html, 2016. Accessed June 1, 2016.

24. Jackevicius CA, Li P, Tu JV. Prevalence, predictors, and outcomes of primary nonadherence after acute myocardial infarction. Circulation. 2008;117(8):1028-1036.
ClinicoEconomics and Outcomes Research

\section{Publish your work in this journal}

ClinicoEconomics and Outcomes Research is an international, peerreviewed open-access journal focusing on health technology assessment, pharmacoeconomics and outcomes research in the areas of diagnosis, medical devices, and clinical, surgical and pharmacological intervention. The economic impact of health policy and health systems
Submit your manuscript here: https://www.dovepress.com/clinicoeconomics-and-outcon-es-rearch-jour

organization also constitute important areas of coverage. The manuscript management system is completely online and includes a very quick and fair peer-review system, which is all easy to use. Visit http://www.dovepress.com/testimonials.php to read real quotes from published authors. 